\title{
Polaron stabilization by cooperative lattice distortion and cation rotations in hybrid perovskite materials
}

Amanda J. Neukirch ${ }^{{ }^{*}}$, Wanyi Nie ${ }^{2}$, Jean-Christophe Blancon ${ }^{3}$ Kannatassen Appavoo $^{4}$, Hsinhan Tsai ${ }^{2}$, Matthew Y. Sfeir ${ }^{4}$, Claudine Katan ${ }^{5}$, Laurent Pedesseau ${ }^{6}$, Jacky Even $^{6}$, Jared J. Crochet $^{2}$, Gautam Gupta ${ }^{2}$,Aditya D. Mohite ${ }^{2}$, and Sergei Tretiak ${ }^{l^{*}}$

${ }^{1}$ Los Alamos National Laboratory, Theoretical Physics and Chemistry of Materials, Los Alamos, NM 87545, USA

${ }^{2}$ Los Alamos National Laboratory, Materials Physics and Application, Los Alamos, NM 87545, USA

${ }^{3}$ Los Alamos National Laboratory, Physical Chemistry and Applied Spectroscopy Division, Los Alamos, NM 87545, USA

${ }^{4}$ Brookhaven National Laboratory, Center for Functional Nanomaterials, Upton, NY 11973, USA

${ }^{5}$ Institut des Sciences Chimiques de Rennes, ISCR, CNRS, Université de Rennes 1, 35042 Rennes, France

${ }^{6}$ Fonctions Optiques pour les Technologies de l'Information, FOTON UMR 6082, CNRS, INSA de Rennes, 35708 Rennes, France

KEYWORDS organic-inorganic perovskite, polaron, photovoltaic, cation rotations 


\section{ABSTRACT}

Solution-processed organometallic perovskites have rapidly developed into a top candidate for the active layer of photovoltaic devices. Despite the remarkable progress associated with perovskite materials, many questions about the fundamental photophysical processes taking place in these devices, remain open. High on the list of unexplained phenomena are very modest mobilities despite low charge carrier effective masses. Moreover, experiments elucidate unique degradation of photocurrent affecting stable operation of perovskite solar cells. These puzzles suggest that while ionic hybrid perovskite devices may have efficiencies on par with conventional Si and GaAs devices, they exhibit more complicated charge transport phenomena. Here we report the results from an in-depth computational study of small polaron formation, electronic structure, charge density and reorganization energies using both periodic boundary conditions and isolated structures. Using the hybrid Density Functional Theory, we found that volumetric strain in a $\mathrm{CsPbI}_{3}$ cluster creates a polaron with binding energy of around $300 \mathrm{meV}$ and $900 \mathrm{meV}$ for holes and electrons, respectively. In the $\mathrm{MAPbI}\left(\mathrm{MA}=\mathrm{CH}_{3} \mathrm{NH}_{3}\right)$ cluster, both volumetric strain and MA reorientation effects lead to larger binding energies at around $600 \mathrm{meV}$ and $1300 \mathrm{meV}$ for holes and electrons, respectively. Such large reorganization energies suggest appearance of small polarons in organometallic perovskite materials. The fact that both volumetric lattice strain and MA molecular rotational degrees of freedom can cooperate to create and stabilize polarons, indicates that in order to mitigate this problem, formamidinium $\left(\mathrm{FA}=\mathrm{HC}\left(\mathrm{NH}_{2}\right)_{2}\right)$ and cesium $(\mathrm{Cs})$ based crystals and alloys, are potentially better materials for solar cell and other optoelectronic applications. 
Hybrid organometallic perovskite (HOP) based solar cells have recently emerged as one of the most promising thin-film photovoltaic materials. ${ }^{1-3}$ For example, solar cells prepared using low-temperature solution-based methods with methyl ammonium $\left(\mathrm{MA}=\mathrm{CH}_{3} \mathrm{NH}_{3}\right)$ lead iodide, $\mathrm{MAPbI}_{3}$, have achieved an increase in efficiency from $3.5 \%$ to over $20 \%$ in about 5 years of effort. ${ }^{4-6}$ The major draw of HOP materials comes from a combination of their high efficiency, low processing cost, and composition from earth-abundant and readily available elements. The unprecedented progress of HOP cell efficiency is indebted to research accomplishments in our understanding of photophysics of other electronically functional nanostructured materials such as organic semiconductors, metalorganic complexes, quantum dots, etc.

Despite the rapid development of organic-inorganic perovskite solar cells, there remain many questions about the fundamental photophysical processes taking place in these devices. For instance, HOP materials are known to have very low effective masses for electrons and holes, at $\sim 0.15 \mathrm{~m}_{0}$ and $0.1 \mathrm{~m}_{0}$ respectively (here $\mathrm{m}_{0}$ is the mass of a free electron), ${ }^{7-10}$ and very low electron-hole recombination rates, ${ }^{11}$ but thin films exhibit modest charge carrier mobilities as compared to conventional semiconductors. ${ }^{12,13}$ In order to capitalize on perovskite materials innate advantageous properties, a comprehensive understanding of the occurring phenomena is required. Just as HOP materials combine the efficiency of conventional semiconductor solar cells with the affordability of organic devices, lessons learned from both inorganic and organic materials are going to have to be integrated into one narrative.

Formation of polaron quasiparticles could prove to be very important for describing the charge carrier behavior in ionic and highly polar crystals such as perovskites. Lately many authors have proposed that the motion of the organic cations, specifically the reorientation of the dipole associated with $\mathrm{CH}_{3} \mathrm{NH}_{3}{ }^{+}$, can stabilize and localize charge carriers in the HOP lattice across 
many unit cells. ${ }^{14-19}$ Electrons self-trapped over several lattice sites in a polarizable medium can be modeled in the effective mass approximation and are known as large polarons ${ }^{20-22}$ Large polarons typically appear in ionic or highly polar crystals (such as many III-V, II-VI semiconductors, alkali halides, and others), ${ }^{22-24}$ where the Coulomb interaction between a conduction electron and the lattice ions results in an enhanced electron-phonon coupling. These quasiparticles have effective sizes much larger than the bond-length and travel through a lattice as free electrons but with an enhanced effective mass, which can explain the observed low electron-hole recombination rates ${ }^{15,25}$ and modest mobility in perovskites ${ }^{18}$ as compared to the values expected from the electron and hole effective mass. Polar fluctuations in HOPs can also support charge carrier separation which would contribute to long carrier lifetimes. ${ }^{26,27}$ Additionally, large polarons have also been invoked to explain light induced changes to the HOP low-frequency dielectric response. ${ }^{28}$

In contrast to the large polaron picture, when the binding energy is large, the effective mass approximation no longer works and the carriers are described as small polarons, which are spatially localized on a few lattice constants. ${ }^{29}$ Small polarons play a very important role in organic semiconductors, ${ }^{30-32}$ and transition metal oxides ${ }^{24,33}$ by controlling charge carrier transport. They may also form in the HOP materials as indirectly evidenced by recent experimental observations of solar cell photodegradation dynamics. ${ }^{34}$ In this case, small polarons would not contribute to the overall carrier mobilities, but are expected to create regions of charge accumulation which in turn cause performance degradation. Other than size, the most important difference between large and small polarons is in their charge transport: large polarons tend to have band-like transport, while small polarons usually undergo incoherent hopping transport. Band and hopping transport are described by different theories and, therefore, in order to 
properly model transport in perovskite materials under different operating conditions, detailed studies on both small and large polaronic effects are needed.

Here we report the results from an in-depth hybrid Density Functional Theory (DFT) study of small polaron formation in HOPs. First, we invoke symmetry analysis in order to determine the lattices degrees of freedom coupled to the electronic system. Periodic boundary condition calculations were then performed to demonstrate how the energy levels and charge density of frontier orbitals were changed by polaron formation. Calculations of isolated clusters were used to explore charged state properties, localization of spin density and to quantitatively evaluate the upper bounds of reorganization energies, which altogether bespeak characteristic small polaron features. To commensurate our computational findings, we finally summarize recent experiments that provide indirect evidence for small polaron formation in a HOP solar devices. We find that upon full vibrational relaxation, small polarons are formed with sizes on the order of a lattice spacing, and binding energy deep into the band gap.

Symmetry considerations: The building blocks of the hybrid lead halide perovskite structure are the $\mathrm{Pb}$ halide octahedra, $\mathrm{PbX}_{6}{ }^{4-}(\mathrm{X}=$ Halide $)$, that are corner-shared to form a $3 \mathrm{D}$ crystalline lattice with $\mathrm{PbX}_{3}^{-}$stoichiometry. The cavities in the inorganic lattice are charge-balanced and filled with organic cations, such as MA= $\mathrm{CH}_{3} \mathrm{NH}_{3}{ }^{+}$. Thus, the crystalline structure of HOPs can be viewed as two alternating sublattices. The VBM (valence band maximum) is mainly the antibonding component of the hybridization between $\mathrm{Pb} s$ states and I $p$ states, while the conduction band minimum $(\mathrm{CBM})$ is dominated by the $\mathrm{Pb} p$ orbitals. Therefore, charge transport in HOPs occurs in the inorganic $\mathrm{PbX}_{3}$ sublattice and the organic $\mathrm{CH}_{3} \mathrm{NH}_{3}{ }^{+}$sublattice acts as a medium that can modify the electrostatic landscape the charge carriers experience, leading to charge screening and localization. ${ }^{18,35}$ 
Electron-phonon coupling is first investigated in the weak coupling regime assuming independent particles allowing group theory analyses. For the reference Pm-3m cubic space group of HOP, the possible intravalley non-polar deformation couplings of the VBM and CBM states only involve acoustic phonons, via a first order acoustic deformation potential (ADP) related to the strain tensor components. ${ }^{36,37}$ Moreover, due to spin-orbit coupling in the $\mathrm{CB}$, only local volumetric strain is expected to couple to VBM and CBM states and influence the first order ADP. This short range interaction is important and may yield in the strong coupling regime the so-called "acoustic polarons", which can trigger self-trapped electronic states. ${ }^{24,38}$ On the other hand, none of the three lattice optical phonons can interact with the VBM and the CBM states via a conventional zero order non-polar optical deformation potential (ODP). ${ }^{36,37}$ Before examining in detail the additional coupling between the VBM and CBM states and the molecular degrees of freedom, let's consider polar coupling mechanisms for phonons. The polar Frölich long range interaction between the delocalized CBM and VBM states and polar optical phonons (FOP) is an important carrier scattering process in hybrid perovskites as a result of dielectric increment between the high and medium frequency ranges related to tongitudinal optical modes. In the strong coupling regime, it yields the so-called "Fröhlich polarons". The classical piezoelectric electron-acoustic phonon polar coupling (PZA) vanishes in Pm-3m crystal structures. Simultaneous vanishing ODP and PZA electron phonon coupling mechanisms is a unique feature, which shows that halide perovskites define a specific new class of semiconductors.

In the parent cubic phase, the MA ion lies at the center of the regular cube formed by eight $\mathrm{Pb}^{2+}$ ions. This requires the MA ion to be disordered in a certain way ${ }^{39}$ Discrete pseudospins (PS) models A, B, and C, Figure 1a-c, correspond to the thermally activated molecular tumbling 
occurring between the 6,8 , and 12 equivalent orientations from the center toward the faces ([100]), the edges ([110]), and the corners ([111]) of the cubic cell respectively. PS are simplified pictures of a more general approach involving continuous rotator functions. ${ }^{40,41}$ Pseudospins and rotator functions allow describing molecular orientational fluctuations via non polar elastic and polar electric multipoles. Elastic multipoles span the same irreducible representations (IR) as the strain components, yielding a linear coupling with the lattice strain. ${ }^{40,41}$ In the strong coupling regime, the formation of polaronic states is thus expected through deformation potentials and short-range interactions, between local volumetric strain, and local molecular arrangements once translational symmetry is lost, Figure $1 \mathrm{~d}$,e. Static orientations of the cations correspond to a superposition of electric and elastic contributions. ${ }^{41}$ Between knowledge of the charge density of band edge states and an understanding of symmetry allowed molecular arrangements lay the ground work for our understanding of the atomistic formation of polarons.

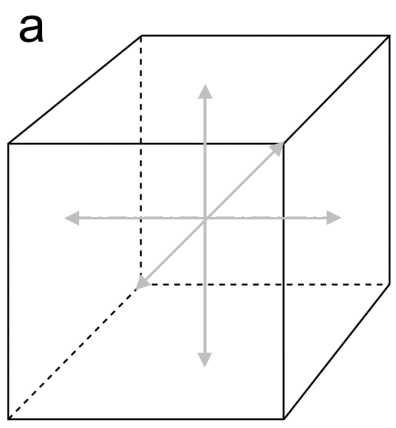

d

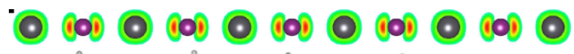

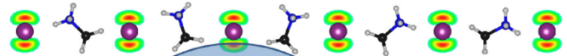
(-) $100001000(001)(100)(100)$ 84848 \& 8 \& 8 \%

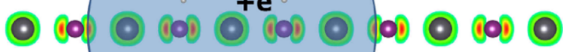
8 \& 8 \& 8 \& 8 म 8

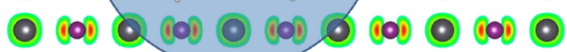

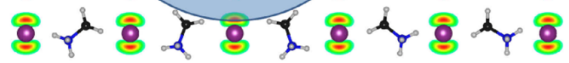

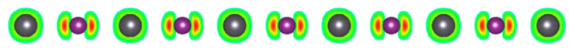
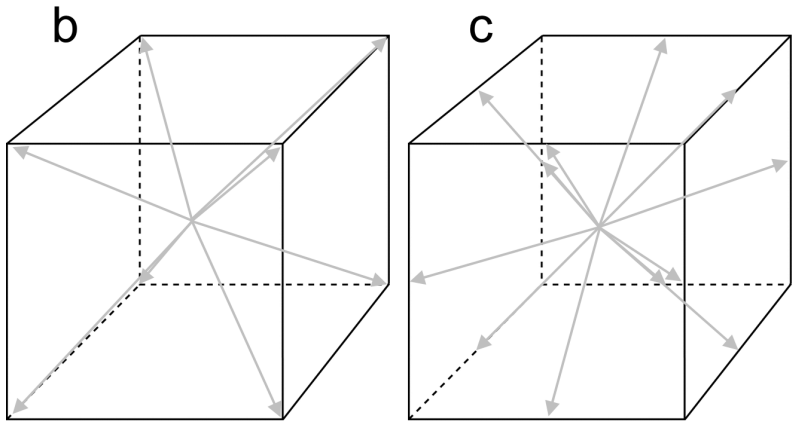

e

(-) 1000 (1000) 10010100101000 8. 8481283.48 (-) 1000 (100) O 1000 (100) 010010

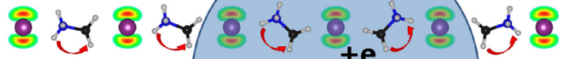

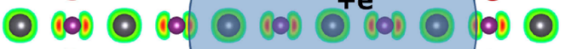

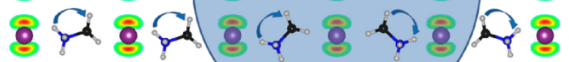

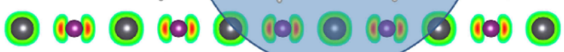

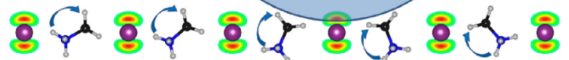
- 1001 (1) 1001 (2) 100101001010010 
Figure 1. a-c Schematic representation of the totally symmetric IR of $\Gamma_{1}^{+}$for the three pseudospins corresponding to [100], [111], and [110] respectively. ${ }^{39}$ Arrows indicate the orientations of $\mathrm{C}-\mathrm{N}$ axis. d, e Artist view of a quasiparticle hole dressed by the interaction with neighboring rotating cations and travelling across the crystal.

Periodic boundary condition (PBC) DFT calculations were initially used to characterize the influence of local lattice deformations and eventual polaron formation. Early PBC calculations using DFT at the Generalized Gradient Approximation (GGA) level showed good agreement between theoretically determined band gaps and experimental measurements in the $\mathrm{MAPbI}_{3}$ material. However, this agreement was due to the cancellation of errors between absence of spin orbit coupling (SOC) and many-body effects. ${ }^{36,42}$ When both phenomena are included (e.g., with computationally expensive GW and SOC frameworks), the good agreement returns. ${ }^{7,8}$ Hybrid functionals that include a fraction of the long-range exact exchange, such as $\mathrm{HSE}^{43}$ generally increase the band gap compared to standard GGA methods, thus improving agreement with experiment. Thus hybrid DFT has been proved to be numerically efficient method resulting in a reasonable accuracy when modeling perovskite materials. ${ }^{8}$ In this work, for calculations involving PBC, we use the HSE and PBE0 functionals including SOC effects as implemented in the Vienna Ab initio Simulation Package (VASP) ${ }^{44,45}$ (see Computational Details section in the SI).

Our modeling starts from the bulk structure for the low temperature orthorhombic phase of $\mathrm{MAPbI}_{3}$ and optimizing until forces are below $0.02 \mathrm{eV} / \AA{ }^{46}$ Based on the above mentioned symmetry analysis, suitable models can be constructed to illustrate the formation of polarons. To this end, to illustrate modification of frontier orbitals due to organic cation motions we consider a neutrally charged super cell of 192 atoms. Consistently with electrostatic interactions, the cationic MA's are rotated towards an electron residing at one of the $\mathrm{Pb}$ atoms mimicking the creation of a 'heavy' charge carrier or small polaron. The nearest neighbor MA cations are first 
manually rotated so that the dipoles are facing towards the chosen $\mathrm{Pb}$ atom. The system is then allowed to optimize for several optimization steps to reduce strain while retaining the direction of the MA dipoles.

Figure 2 shows the change in VBM and CBM states from the unperturbed system (a,b) and when the nearest 8 MA dipoles are rotated towards the $\mathrm{Pb}$ atom $(\mathrm{c}, \mathrm{d})$. In this case the charge density of the VBM remains highly delocalized away from the central $\mathrm{Pb}$ atom, while that of the $\mathrm{CBM}$ becomes localized on that $\mathrm{Pb}$ cation. This is indicative of the creation of a polaron for an excited electron in the CBM. The observed separation of charges is going to reduce radiative recombination between mobile and localized carriers. It should be noted that the charge densities remain unchanged with respect to cut-off energy, inclusion of SOC, van der Waal's interactions, and between functionals. In order to obtain a first estimate of the polaron stabilization energy, we calculated the change in band gap between the unperturbed system and the system where MA's have been rotated. Using both the HSE and PBE0 functional we got a band gap stabilization of about $160 \mathrm{meV}$ in both cases.

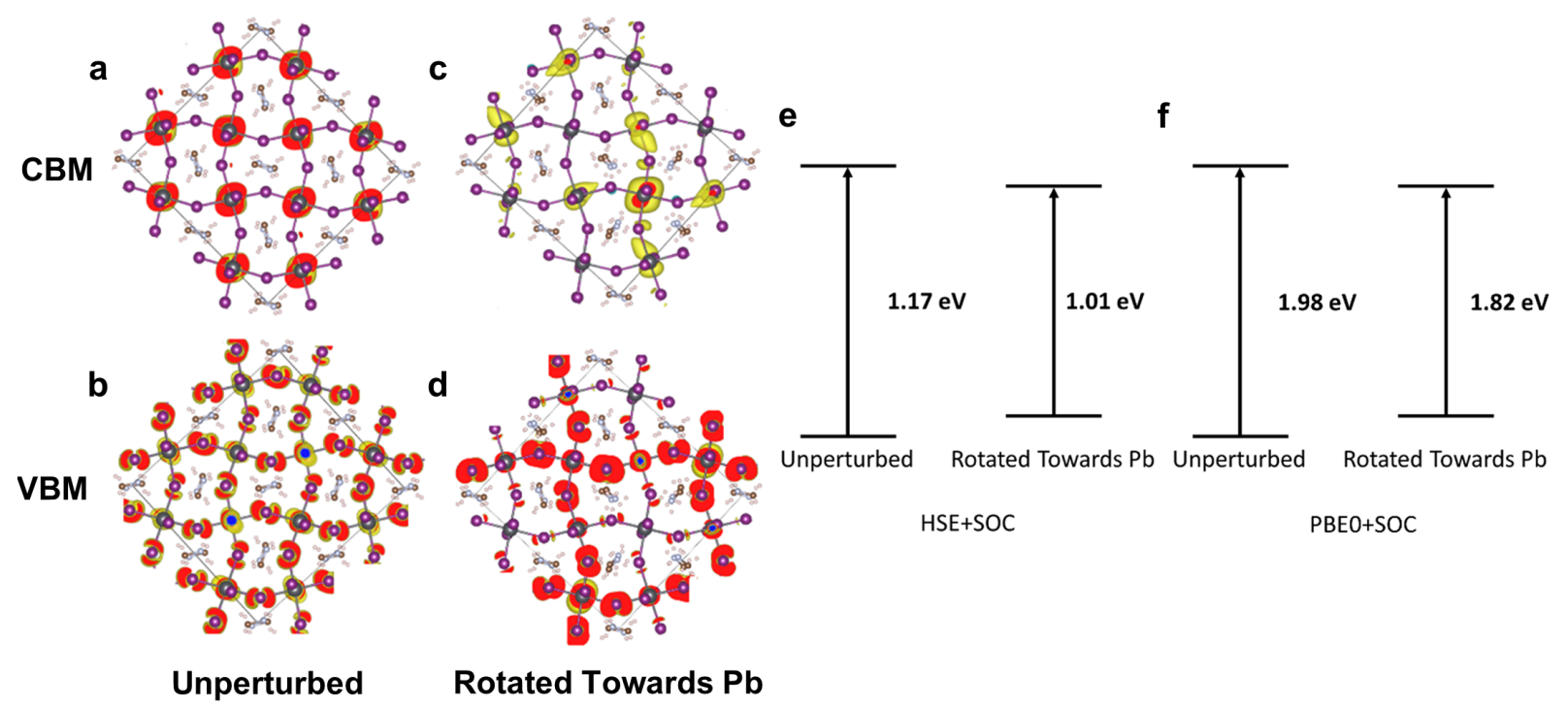

Figure 2 Localization due to rotation of MA cations. Crystal structures and charge densities including SOC of VBM and CBM a, b optimized experimental structure, $\mathbf{c}, \mathbf{d}$ when 8 MA's are 
rotated towards a specific $\mathrm{Pb}$ atom (right panel). Variation of the band gap energy computed at the HSE+SOC (e) and PBE0+SOC (f) levels of theory for the unperturbed structure and the structure where $8 \mathrm{MA}$ 's are rotated towards a specific $\mathrm{Pb}$ atom.

Isolated structure calculations: PBC, however, do not allow treatment of charged states due to spurious interaction of the charged cell with its replicas. We subsequently conducted simulations of finite structures similar to what has been done in the case of organic semiconductors. ${ }^{30-32}$ When simulating charged systems, long range interactions are expected to play a significant role, requiring range-corrected functionals. For example, the hybrid exchange-correlation CAMB3LYP functional (here fraction of the exact exchange varies from $20 \%$ to $65 \%)^{47}$ in the presence of a polarizable solvent, reproduces correctly the polaronic effects in the organic semiconductors. ${ }^{30-32}$ In this work, all calculations of HOP isolated clusters were performed using the CAM-B3LYP functional combined with the LANL2dz (for Pb and I) and 6-31G* (for N, C, and $\mathrm{H}$ ) basis sets using Gaussian 09 software package ${ }^{48}$ (see Computational Details section in the SI). Additionally, we include a polar solvent $(\varepsilon=78)$ via the conductor-like polarizable continuum model. ${ }^{49}$ For comparison between the periodic system and isolated clusters, the calculations were also performed using the HSE model and these results are shown in the SI.

There were several considerations when designing the isolated HOP structures. We started with lattice parameters that were derived from variable temperature powder x-ray diffraction experiments, taking the low temperature structure of $\mathrm{MAPbI}_{3}$ as refined in a cubic lattice. ${ }^{46} \mathrm{In}$ order to minimize dangling bonds, the cluster should be terminated on all sides with the MAI (CsI). There must also be charge balance. Eqs. 1 and 2 ensure that the system is overall neutral

$$
\begin{gathered}
6 n_{P b}=2 n_{I_{1}}+n_{I_{2}} \\
2 n_{P b}+n_{M A}=n_{I_{1}}+n_{I_{2}}
\end{gathered}
$$


Here $n_{P b}, n_{M A}$, and $n_{I}$ are the number of $\mathrm{Pb}, \mathrm{MA}$, and I atoms respectively. $I_{1}$ corresponds to an atom on the surface that is only bonded to one $\mathrm{Pb}$ atom, while $I_{2}$ corresponds to an $\mathrm{I}$ atom that is bonded to two $\mathrm{Pb}$ atoms. The cluster must be large enough to include at least $8 \mathrm{MA}$ cations surrounding the central $\mathrm{Pb}$ atom. To fulfill all conditions, we end up with a cluster having the following stoichiometry: $\mathrm{MA}_{54} \mathrm{~Pb}_{27} \mathrm{I}_{108}\left(\mathrm{I}_{1}=\mathrm{I}_{2}=54\right)$. A cube fully terminated by $\mathrm{MA}(\mathrm{Cs})$ and $\mathrm{I}$ would require $64 \mathrm{MA}$ molecules. In order to achieve charge balance between the ions and maximize symmetry the 8 corner MA's were removed as were two opposite MA molecules on opposite edges. Figure $\mathrm{S} 1$ in the SI shows the resulting structures for both $\mathrm{MA}_{54} \mathrm{~Pb}_{27} \mathrm{I}_{108}$ and $\mathrm{Cs}_{54} \mathrm{~Pb}_{27} \mathrm{I}_{108}$ isolated clusters before and after geometry optimization. Despite the change in stoichiometry because the clusters originate from the bulk 3D geometry we will still refer to them as $\mathrm{MAPbI}_{3}$ and $\mathrm{CsPbI}$.

Performing calculations with both $\mathrm{Cs}$ and MA cations allows for separation between the effects of dipole rotation and volumetric strain. We will start by summarizing what takes place in the Cs-based system. The procedure for determining the polaron binding energy is to optimize the unperturbed neutral system. Once the geometry has been completely optimized, we add/remove an electron and perform a single point calculation to get the energy of the charged system in the neutral ground state geometry. We then compare this energy to a Cs-based system that has been geometrically optimized while charged.

Figure 3 illustrates the spin density of electrons and holes in the optimized ground state and charged geometries. Of note is that even optimization of the ideal structure in the neutral states causes some charge localization in the charged state, emphasizing that by studying isolated clusters we are providing an upper bound for polaron binding energy. When the atomic coordinates are optimized in the respective charged states, holes show only a slight increase in 
their localization while electrons display a dramatic localization. Since an electron in the bottom of the conduction band is predominantly located on $\mathrm{Pb}$ atoms, and the cell we designed has $\mathrm{Pb}$ at the center, it is not surprising that the electron is the species that preferentially forms a polaron. It should be noted that a hole on a central halide would repel both the surrounding $\mathrm{Pb}$ and $\mathrm{Cs} / \mathrm{MA}$ cations, reducing polaron binding energy. Using the HSE functional Figure S2 in the SI, both the electron and hole spin density in the ground state geometry are delocalized across the entire cluster. For the charged geometries, the hole remains delocalized while the electron becomes noticeably localized.

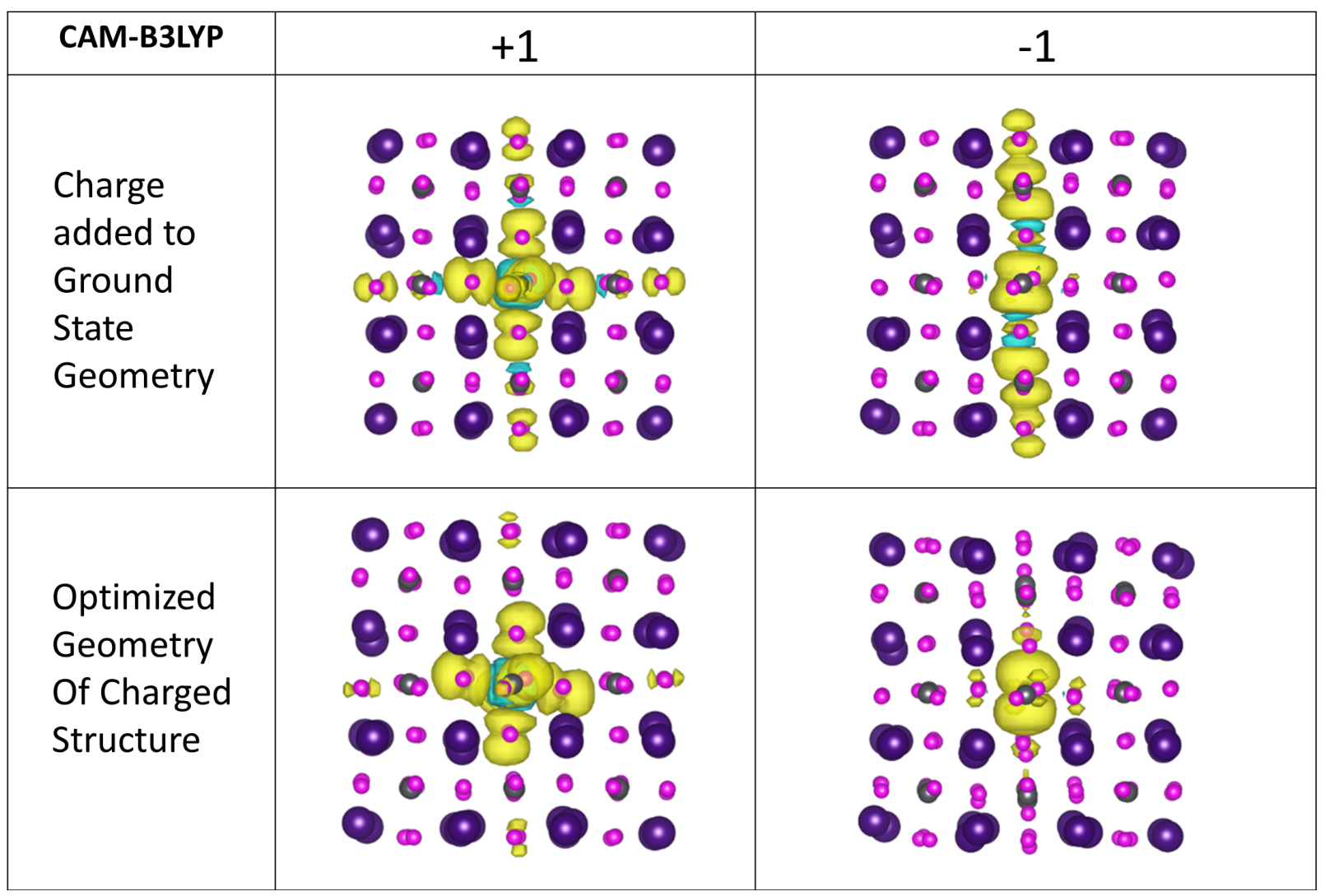

Figure 3 Hole (left) and electron (right) spin density distribution of optimized neutral (top) and charged (bottom) $\mathrm{CsPbI}_{3}$ clusters calculated using the CAM-B3LYP functional.

In order to quantify the polaron binding energy we calculate the two relaxation energies, the sum of which is the reorganization energy as, for example, enters in a standard Marcus theory 
describing electron transfer, ${ }^{50}$ as shown in Figure S3 in the SI. The internal reorganization energy essentially quantifies variation of electronic energy due to the geometry changes when an electron is added or removed from the cluster. ${ }^{51}$ The intramolecular reorganization energy is the sum of two components $\left(\lambda_{1}\right.$ and $\left.\lambda_{2}\right)$ defined as

$$
\lambda_{\text {reorg }}=\lambda_{1}+\lambda_{2}=\left(E^{*}-E\right)+\left(E_{c}^{*}-E_{c}\right)
$$

Where $\lambda_{1}$ represents the change in energy between the neutral species in the optimized geometry of charged state $\left(E^{*}\right)$ and the relaxed neutral species $(E)$. The respective energy difference for the charged system at the neutral species geometry $\left(E_{c}^{*}\right)$ and the relaxed charge geometry $\left(E_{c}\right)$, is represented by $\lambda_{2}$. The polaron binding energy is given by $\lambda_{2}$. Using the CAM-B3LYP functional, we found that electrons have a binding energy of about $900 \mathrm{meV}$, which is much larger than the $270 \mathrm{meV}$ binding energy for holes, Table 1. The results for the HSE functional are given in Table S1 and Figure S4 in the SI. As expected, binding energies for both holes and electrons decreased to $36 \mathrm{meV}$ and $398 \mathrm{meV}$, respectively, owing to the lack of appropriate electronic exchange. Notably, semilocal models (e.g., GGA) and hybrid functionals with a small fraction of exact exchange (e.g., B3LYP, PBE0, HSE) are unable to describe polarons in other systems such as organic semiconductors. ${ }^{30-32}$ As a reminder, all these numbers serve as an upper bound in polaron binding energy as the introduction of a finite system introduces quantum confinement effects.

Table 1 Reorganization energies, in $\mathrm{meV}$, for charged $\mathrm{CsPbI}_{3}$ and $\mathrm{MAPbI}_{3}$ clusters. Schematic representation of internal reorganization energy given in Fig. S3 in SI.

\begin{tabular}{c|ccc|ccc}
\hline \multirow{2}{*}{ CAM-B3LYP } & \multicolumn{3}{|c|}{ Electron } & \multicolumn{3}{c}{ Hole } \\
\cline { 2 - 7 } & $\lambda_{1}$ & $\lambda_{2}$ & $\lambda$ & $\lambda_{1}$ & $\lambda_{2}$ & $\lambda$ \\
\hline Cs & 724 & 918 & 1642 & 365 & 274 & 639 \\
MA & 510 & 1377 & 1887 & 528 & 578 & 1106
\end{tabular}


Moving on to the $\mathrm{MAPbI}_{3}$ system, the procedure for finding the binding energy changes slightly. Once the neutral geometry has been optimized and a single point energy has been calculated for the charged versions, this energy is compared to a system where the MA molecules have been rotated (towards the central $\mathrm{Pb}$ atom for electrons and away for holes) and geometrically optimized in their charged state, as shown Figure 4 for the CAM-B3LYP functional. This ensures better starting structures for geometry relaxation of the charged states based on physical intuition. In the neutral geometry the hole electronic density is not in the center. This occurs because the geometry optimization spontaneously breaks the cluster symmetry and/or because the hole prefers to be centered on a halide which is not going to be centrally located. However in both cases the electron and the hole are significantly localized once the system is optimized in the charged state.

Electrons form a polaron binding energy of $1377 \mathrm{meV}$ as given by the CAM-B3LYP model, Table 1. The magnitude of the polaron binding energy is likely over-estimated due to confinement effects, but it is clear that tighter polarons are formed when MA atoms are present. The binding energy for holes is $578 \mathrm{meV}$. It should be made clear that not all of the binding energy comes from contributions of MA rotations as the system is also able to undergo volumetric strain during the optimization process. When these calculations are performed using the HSE functional (Table S1) the binding energies for holes and electrons become 33 and 880 meV respectively. The fact that both volumetric $(\mathrm{Cs})$ and rotational strain $(\mathrm{MA})$ can cooperate to small polaron formation indicates that the best way to reduce polaron formation, would be to use a larger organic cation with a smaller dipole moment such as Formamidinium $\left(\mathrm{FA}=\mathrm{HC}\left(\mathrm{NH}_{2}\right)_{2}\right)$. These theoretical and experimental insights could explain why the current top perovskite solar cells are made with alloys containing FA. ${ }^{6,52-54}$ Recent reports on perovskite cells demonstrate 
that mixed alloys involving MA, FA, and Cs open the road toward further improvements, both for cell efficiency and stability. ${ }^{6,52-55}$

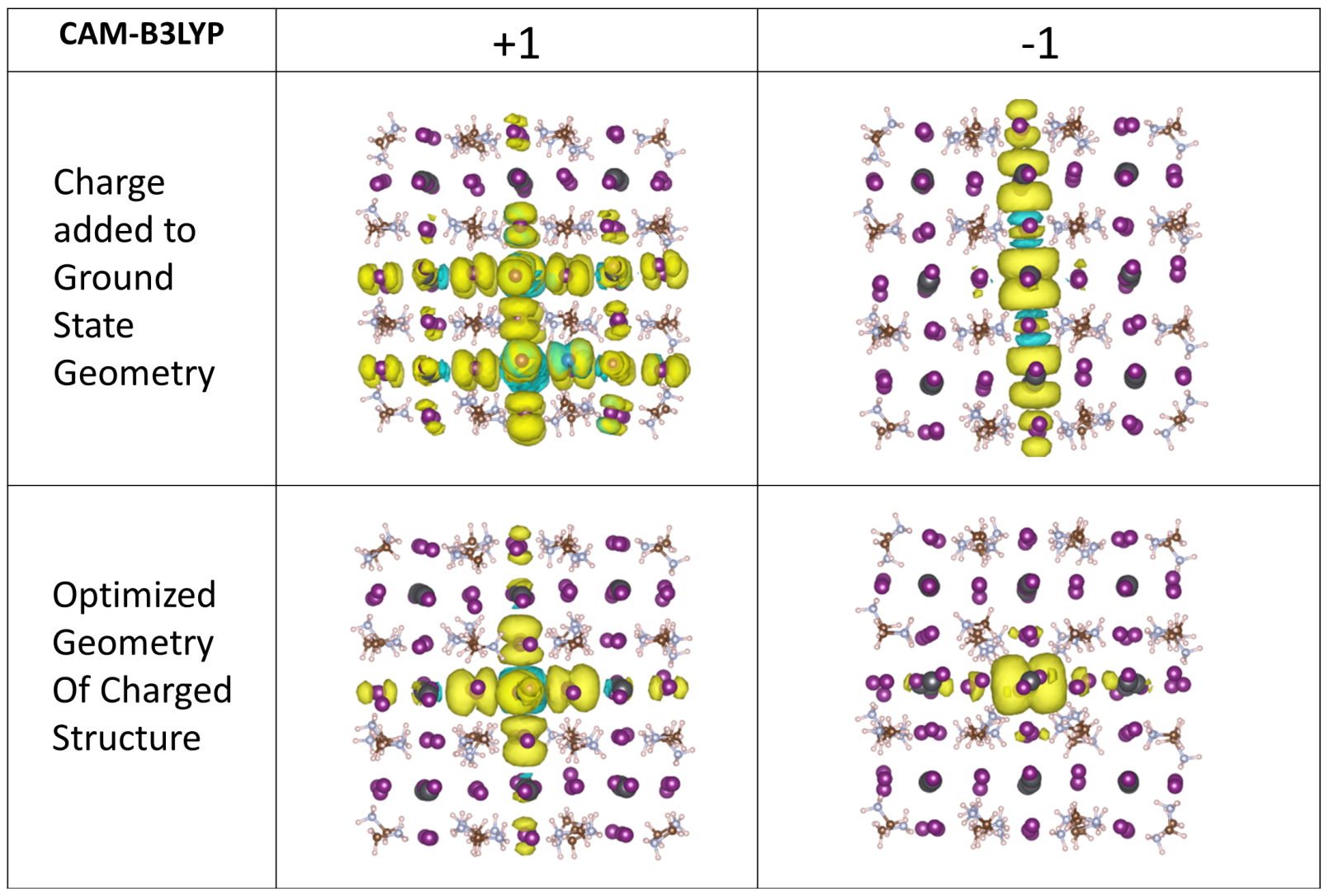

Figure 4 Hole (left) and electron (right) spin density distribution of optimized neutral (top) and charged (bottom) $\mathrm{MAPbI}_{3}$ clusters calculated using the CAM-B3LYP functional.

Experimental Evidence: We recently demonstrated the reduction in photocurrent, and subsequently device efficiency, in crystalline large grain size $\mathrm{MAPbI}_{3}$ perovskite solar cells under constant solar illumination. The efficiency rapidly recovers to its original value after less than 1 minute in the dark. ${ }^{34}$ Localized charged states strongly coupled to local structural lattice distortions and MA quasistatic configurations creating small polaron states, offer a nice explanation for the experimental observation of very slow degradation and fast self-healing on perovskite cell devices. In this picture, small polarons produced by light irradiation seed the formation of macroscopic charged domains preventing efficient charge extraction. 
Conclusion: Polaron quasiparticles frequently determine the charge carrier behavior in ionic and highly polar crystals such as perovskites. In this manuscript our computational study suggest small polaron formation in HOPs. A thorough investigation shows that localized charged states strongly couple to local structural lattice distortions and MA quasistatic configurations. Through symmetry arguments and DFT calculations using hybrid functionals, it was shown that MA dipoles can align with a charge centered on a $\mathrm{Pb}$ atom. Periodic boundary condition calculations demonstrate that frontier orbitals become localized after MA reorientation and that the band gap shows a stabilization in the 100's of meV. Moving on to charged isolated clusters, we found that electrons have a polaron binding energy much larger than holes, and are therefore able to predict that electrons are more readily trapped. Using the CAM-B3LYP functional we found that volumetric strain in a $\mathrm{CsPbI}_{3}$ cluster creates a polaron with binding energy of around $900 \mathrm{meV}$. In the $\mathrm{MAPbI}_{3}$ cluster there is both volumetric strain and $\mathrm{MA}$ reorientation so binding energies are deeper at around $1300 \mathrm{meV}$. These computational insights allow rationalizing experimental studies showing that prolonged exposure to light leads to the accumulation of space charge, and yet there is no physical degradation or change in stoichiometry of the sample. Additionally, there is an evidence of a slowdown in MA rotation and formation of light-activated metastable trap states that are localized more than $500 \mathrm{meV}$ deep within the band gap ${ }^{34}$ in excellent agreement with our numerical results. Such comprehensive computational studies are necessary in order to fully understand the complex behavior of perovskite materials and maximize electronic properties of these interesting materials to their full potential. For example, the fact that both lattice volumetric strain and rotational degrees of freedom can cooperate to create polarons, justifies superior performance of $\mathrm{FAPbI}_{3}$ and Cs containing materials in solar cell applications. ${ }^{6,55}$ 


\section{ASSOCIATED CONTENT}

Supporting Information Available: Computational details and structures for Cs and MA isolated clusters before and after optimization, structures of isolated clusters, HSE charge densities for $\mathrm{CsPbI}$ and $\mathrm{MAPbI}_{3}$, schematic representation of reorganization energy, and experimental evidence for polarons.

This material is available free of charge via the Internet at http://pubs.acs.org.

\section{AUTHOR INFORMATION}

\section{Corresponding Authors}

*E-mail: ajneukirch@lanl.gov; serg@lanl.gov

\section{Author Contributions}

All authors have given approval to the final version of the manuscript.

\section{Funding Sources}

The work at Los Alamos National Laboratory (LANL) was supported by the LANL LDRD program (A.J.N., A.D.M, G.G and S.T.). This work was done in part at Center for Nonlinear Studies (CNLS) and the Center for Integrated Nanotechnologies CINT), a U.S. Department of Energy and Office of Basic Energy Sciences user facility, at LANL. This research used resources provided by the LANL Institutional Computing Program. LANL is operated by Los Alamos National Security, LLC, for the National Nuclear Security Administration of the U.S. Department of Energy under contract DE-AC52-06NA25396. The work in France was supported by Cellule Energie du CNRS (SOLHYBTRANS Project) and University of Rennes 1 
(Action Incitative, Défis Scientifique Emergents 2015). J.E. work was supported by the Fondation d'entreprises banque Populaire de l'Ouest under Grant PEROPHOT 2015.

\section{ACKNOWLEDGMENT}

AJN would like to thank Mikael Kepenekian for useful insight and discussion on the set up of the calculations.

\section{REFERENCES}

(1) Mei, A.; Li, X.; Liu, L.; Ku, Z.; Liu, T.; Rong, Y.; Xu, M.; Hu, M.; Chen, J.; Yang, Y.; Gratzel, M.; Han, H. Science (80-. ). 2014, 345 (6194), 295-298.

(2) Lee, M. M.; Teuscher, J.; Miyasaka, T.; Murakami, T. N.; Snaith, H. J. Science 2012, 338 (6107), 643-647.

(3) Sheng, R.; Ho-Baillie, A.; Huang, S.; Chen, S.; Wen, X.; Hao, X.; Green, M. A. J. Phys. Chem. C 2015, 119 (7), 3545-3549.

(4) Kojima, A.; Teshima, K.; Shirai, Y.; Miyasaka, T. 2009, 6050-6051.

(5) Im, J.; Lee, C.; Lee, J.; Park, S.; Park, N. 2011, 2, 4088-4093.

(6) Yang, W. S.; Noh, J. H.; Jeon, N. J.; Kim, Y. C.; Ryu, S.; Seo, J.; Seok, S. Science (80-. ). 2015, No. 348, 1234.

(7) Umari, P.; Mosconi, E.; De Angelis, F. Sci. Rep. 2014, 4, 4467.

(8) Menendez-Proupin, E.; Palacios, P.; Wahnon, P.; Conesa, J. C. Phys. Rev. B 2014, 90, 045207.

(9) Miyata, A.; Mitioglu, A.; Plochocka, P.; Portugall, O.; Wang, J. T.-W.; Stranks, S. D.; Snaith, H. J.; Nicholas, R. J. Nat Phys 2015, 11 (7), 582-587.

(10) Galkowski, K.; Mitioglu, A.; Miyata, A.; Plochocka, P.; Portugall, O.; Eperon, G. E.; Wang, J. T.-W.; Stergiopoulos, T.; Stranks, S. D.; Snaith, H. J.; Nicholas, R. J. Energy Environ. Sci. 2016, 9 (3), 962-970.

(11) Blancon, J.; Nie, W.; Neukirch, A. J.; Gupta, G.; Tretiak, S.; Cognet, L.; Mohite, A. D.; Crochet, J. J. Adv. Funct. Mater. 2016. doi: 10.1002/adfm.201505324 (in press).

(12) Brenner, T. M.; Egger, D. A.; Rappe, A. M.; Kronik, L.; Hodes, G.; Cahen, D. J. Phys. Chem. Lett. 2015, 6 (23), 4754-4757.

(13) Brenner, T. M.; Egger, D. A.; Kronik, L.; Hodes, G.; Cahen, D. Nat. Rev. Mater. 2016, 1, 15007.

(14) Walsh, A. J. Phys. Chem. C 2015, 119 (11), 5755-5760.

(15) Ma, J.; Wang, L.-W. Nano Lett. 2014, 15 (1), 248-253. 
(16) Quarti, C.; Mosconi, E.; De Angelis, F. Phys. Chem. Chem. Phys. 2015, 17 (14), 93949409.

(17) Carignano, M. A.; Kachmar, A.; Hutter, J. J. Phys. Chem. C 2015, 9019, 150304155542004.

(18) Zhu, X. Y.; Podzorov, V. J. Phys. Chem. Lett. 2015, 6 (23), 4758-4761.

(19) Kamat, P. J. Phys. Chem. Lett. 2015, 6 (23), 4874-2875.

(20) Feynman, R. Phys. Rev. 1955, 97 (3), 660-665.

(21) Fröhlich, H. Adv. Phys. 1954, 3 (11), 325-361.

(22) Devreese, J. T.; Alexandrov, A. S. Reports Prog. Phys. 2009, 72, 066501.

(23) Devreese, J. T. J. Phys. Condens. Matter 2007, 19, 255201.

(24) Shluger, A.; Stoneham, A. J. Phys. Condens. Matter 1993, 5 (19), 3049-3086.

(25) Zheng, F.; Tan, L. Z.; Liu, S.; Rappe, A. M. Nano Lett. 2015, 15 (12), 7794-7800.

(26) Frost, J. M.; Butler, K. T.; Brivio, F.; Hendon, C. H.; Van Schilfgaarde, M.; Walsh, A. Nano Lett. 2014, 14 (5), 2584-2590.

(27) Liu, S.; Zheng, F.; Koocher, N. Z.; Takenaka, H.; Wang, F.; Rappe, A. M. J. Phys. Chem. Lett. 2015, 6 (4), 693-699.

(28) Juarez-Perez, E. J.; Sanchez, R. S.; Badia, L.; Garcia-Belmonte, G.; Kang, Y. S.; MoraSero, I.; Bisquert, J. J. Phys. Chem. Lett. 2014, 5 (13), 2390-2394.

(29) Holstein, T. Ann. Phys. (N. Y). 1959, 389, 343-389.

(30) Nayyar, I. H.; Batista, E. R.; Tretiak, S.; Saxena, A.; Smith, D. L.; Martin, R. L. J. Phys. Chem. Lett. 2011, 2 (6), 566-571.

(31) Nayyar, I. H.; Batista, E. R.; Tretiak, S.; Saxena, A.; Smith, D. L.; Martin, R. L. J. Chem. Theory Comput. 2013, 9 (2), 1144-1154.

(32) Nayyar, I. H.; Batista, E. R.; Tretiak, S.; Saxena, A.; Smith, D. L.; Martin, R. L. J. Polym. Sci. Part B Polym. Phys. 2013, 51 (12), 935-942.

(33) Muñoz Ramo, D.; Shluger, A. L.; Gavartin, J. L.; Bersuker, G. Phys. Rev. Lett. 2007, 99 (15), 1-4.

(34) Nie, W.; Blancon, J.; Neukirch, A. J.; Appavoo, K.; Tsai, H.; Chhowalla, M.; Alam, M. A.; Sfeir, M. Y.; Katan, C.; Even, J.; Tretiak, S.; Crochet, J. J.; Gupta, G.; Mohite, A. D. Nat. Commun. 2016, 7, 11574.

(35) Even, J.; Pedesseau, L.; Katan, C. J. Phys. Chem. C 2014, 118, 11566.

(36) Even, J.; Pedesseau, L.; Katan, C.; Kepenekian, M.; Lauret, J. S.; Sapori, D.; Deleporte, E. J. Phys. Chem. C 2015, 119 (19), 10161-10177.

(37) Robles, R.; Katan, C.; Sapori, D.; Pedesseau, L.; Even, J.; Chimiques, S.; Uab, C.; Umr, F. ACS Nano 2015, 9 (12), 11557-11567.

(38) Vlietinck, J.; Casteels, W.; Houcke, K. Van; Tempere, J.; Ryckebusch, J.; Devreese, J. T. New J. Phys. 2015, 17 (3), 033023.

(39) Onoda-Yamamuro, N.; Matsuo, T.; Suga, H. J. Phys. Chem. Solids 1992, 53 (7), 935-939. 
(40) Even, J. J. Phys. Chem. Lett. 2015, 6 (12), 2238-2242.

(41) Even, J.; Carignano, M.; Katan, C. Nanoscale 2016, 8, 6222-6236.

(42) Even, J.; Pedesseau, L.; Jancu, J. M.; Katan, C. J. Phys. Chem. Lett. 2013, 4, 2999-3005.

(43) Heyd, J.; Scuseria, G. E. J. Chem. 2004, 121, 1187.

(44) Kresse, G.; Furthmuller, J. Comput. Mater. Sci.1996, 6, 15-50.

(45) Kresse, G.; Joubert, D. Phys. Rev. B 1999, 59, 1758-1775.

(46) Baikie, T.; Fang, Y.; Kadro, J. M.; Schreyer, M.; Wei, F.; Mhaisalkar, S. G.; Gratzel, M.; White, T. J. J. Mater. Chem. A 2013, 1 (18), 5628.

(47) Yanai, T.; Tew, D. P.; Handy, N. C. Chem. Phys. Lett. 2004, 393 (1-3), 51-57.

(48) Frisch, M. J.; Trucks, G. W.; Schlegel, H. B.; Scuseria, G. E.; Robb, M. A.; Cheeseman, J. R.; Scalmani, G.; Barone, V.; Mennucci, B.; Petersson, G. A.; Nakatsuji, H.; Caricato, M.; Li, X.; Hratchian, H. P.; Izmaylov, A. F.; Bloino, J.; Zheng, G.; Sonnenberg, J. L.; Hada, M.; Ehara, M.; Toyota, K.; Fukuda, R.; Hasegawa, J.; Ishida, M.; Nakajima, T.; Honda, Y.; Kitao, O.; Nakai, H.; Vreven, T.; Montgomery Jr., J. A.; Peralta, J. E.; Ogliaro, F.; Bearpark, M.; Heyd, J. J.; Brothers, E.; Kudin, K. N.; Staroverov, V. N.; Kobayashi, R.; Normand, J.; Raghavachari, K.; Rendell, A.; Burant, J. C.; Iyengar, S. S.; Tomasi, J.; Cossi, M.; Rega, N.; Millam, J. M.; Klene, M.; Knox, J. E.; Cross, J. B.; Bakken, V.; Adamo, C.; Jaramillo, J.; Gomperts, R.; Stratmann, R. E.; Yazyev, O.; Austin, A. J.; Cammi, R.; Pomelli, C.; Ochterski, J. W.; Martin, R. L.; Morokuma, K.; Zakrzewski, V. G.; Voth, G. A.; Salvador, P.; Dannenberg, J. J.; Dapprich, S.; Daniels, A. D.; Farkas, Ö.; Foresman, J. B.; Ortiz, J. V; Cioslowski, J.; Fox, D. J. Gaussian Inc. Wallingford CT 2009 2009.

(49) Barone, V.; Cossi, M. J. Phys. Chem. A 1998, 102 (11), 1995-2001.

(50) Bao, Z.; Locklin, J. Organic Field-Effect Transistors; CRC Press, 2007.

(51) Marcus, R. A. Rev. Mod. Phys. 1993, 32 (8), 1111-1121.

(52) Yi, C.; Li, X.; Luo, J.; Zakeeruddin, S. M.; Grätzel, M. Adv. Mater 2016.

(53) McMeekin, D. P.; Sadoughi, G.; Rehman, W.; Eperon, G. E.; Saliba, M.; Horantner, M. T.; Haghighirad, A.; Sakai, N.; Korte, L.; Rech, B.; Johnston, M. B.; Herz, L. M.; Snaith, H. J. Science (80-. ). 2016, 351 (6269), 151-155.

(54) Beal, R. E.; Slotcavage, D. J.; Leijtens, T.; Bowring, A. R.; Belisle, R. A.; Nguyen, W. H.; Burkhard, G. F.; Hoke, E. T.; McGehee, M. D. J. Phys. Chem. Lett. 2016, 746-751.

(55) Saliba, M.; Matsui, T.; Seo, J.-Y.; Domanski, K.; Correa-Baena, J.-P.; Mohammad K., N.; Zakeeruddin, S. M.; Tress, W.; Abate, A.; Hagfeldt, A.; Grätzel, M. Energy Environ. Sci. 2016. 